\title{
THE
}

\section{On the Mixing Enhancement in a Meandering Jet Due to the Interaction with an Eddy}

Stephanie Dutkiewicz

University of Rhode Island

Nathan Paldor

University of Rhode Island

Follow this and additional works at: https://digitalcommons.uri.edu/gsofacpubs

\section{Citation/Publisher Attribution}

Dutkiewicz, S., Y\& Paldor, N. (1994. On the Mixing Enhancement in a Meandering Jet Due to the Interaction with an Eddy. J. Phys. Oceanogr., 24, 2418-2423. doi: 10.1175/1520-0485(1994)0242.0.C0;2. Available at: https://doi.org/10.1175/1520-0485(1994)0242.0.C0;2

This Article is brought to you for free and open access by the Graduate School of Oceanography at DigitalCommons@URI. It has been accepted for inclusion in Graduate School of Oceanography Faculty Publications by an authorized administrator of DigitalCommons@URI. For more information, please contact digitalcommons-group@uri.edu. 


\title{
On the Mixing Enhancement in a Meandering Jet Due to the Interaction with an Eddy
}

\author{
STEPHANiE DUTKIEWICZ AND NATHAN PALDOR* \\ Graduate School of Occanography, University of Rhode Island, Narraganselt, Rhode Island
}

18 February 1993 and 17 February 1994

\begin{abstract}
The interaction between a simple meandering jet, such as the Gulf Stream, and an eddy is shown to greatly enhance the mixing and dispersal of fluid parcels in the jet. This enhanced mixing is quantified by calculating the rate of increase of the root-mean-square pair separation of Lagrangian particles (e.g., floats) launched in the jet's immediate vicinity. In the presence of an eddy, particles can escape from the regions in which they were initially launched. Comparisons with observations show a markedly improved qualitative agreement when the eddy is allowed to interact with the meandering jet.
\end{abstract}

\section{Introduction}

Mixing in frontal zones has been the focus of several recent studies. One such, intensively investigated, frontal zone is the Gulf Stream: an eastward moving jet separating cold slope waters from warm Sargasso Sea water. Mixing across this front will therefore affect the distribution of dynamically important tracers such as temperature and salinity, as well as nutrients and biology in both slope waters and the Sargasso Sea. In an attempt to quantify this mixing, the mechanisms responsible for it have to be isolated. Bower and Rossby (1989) showed that the meanders associated with the Gulf Stream are responsible for much of the crossstream motion of RAFOS floats within the jet. However, meanders alone cannot lead to the motion of particles from one side of the jet to another. We expect that diffusion of properties due to small-scale, incoherent processes is important in that respect and might lead to cross-frontal mixing, but larger-scale events, such as the interaction of the jet with an eddy, might also be essential.

Mixing processes can be studied using simple models. Since the Gulf Stream has a coherent structure that when viewed along isopycnal surfaces appears two-dimensional, we can employ a simple two-dimensional kinematic model, such as Bower (1991). Her model describes a simple streamfunction that reproduces some of the kinematic features of an eastward propagating meandering jet, and in this model the meander parameters affect the

\footnotetext{
* Permanent affiliation: Department of Atmospheric Sciences, The Hebrew University of Jerusalem, Jerusalem, Israel.

Corresponding author address: Ms. Stephanie Dutkiewicz, Graduate School of Oceanography, University of Rhode Island, Narragansett, RI 02882-1197.
}

rate and amount of water that propagate downstream. However, Bower's model does not allow for any mixing, especially the important movement of fluid particles from one side of the jet to another. Dutkiewicz et al. (1993) use a similar model in which a Markovian process is added to the deterministic motion of the particles to simulate the small-scale diffusion expected in the real ocean. Mixing was enhanced and there was movement of water parcels across the jet. Jets such as the Gulf Stream do not remain invariant in shape as meanders constantly grow and diminish. Time dependence of the meander's parameters was used by Samelson (1992) to increase mixing and to allow movement of particles across the front. Another way that the Gulf Stream will change shape, is by interacting with a Gulf Stream ring. The inclusion of an eddy (e.g., a Gulf Stream ring) in the simple meandering jet model should then enhance the mixing of fluid parcels within the jet. This process is the subject of the present note.

Gulf Stream rings are formed when a meander elongates and pinches off from the jet, trapping water from the other side of the jet in its core. Thus, rings will form anomalous property signals on either side of the jet-warm core rings in the cold slope water and cold core rings on the warm Sargasso side. These features maintain their structure and move slowly westward often interacting with the jet further upstream. Such an interaction will cause changing flow patterns in the jet, as shown by modeling studies such as Nof (1986). Rings can be reabsorbed and detach again, as seen in some of the model runs by Spall and Robinson (1990). Gulf Stream jet-eddy interactions have been observed to enhance fluid exchange across the jet's boundaries (Bower and Rossby 1989). In both their dynamical and kinematic models, Dewar and Flierl (1985) showed that eddies tend to increase the rate of dispersion of fluid particles, especially in the along-jet direction. The 
Gulf Stream frequently interacts with many rings and it can be expected that these eddies play an essential role in determining the distribution of tracer properties in the vicinity of the stream (Olson 1991).

This note considers a very simplified model of a twodimensional kinematic jet-eddy interaction. Our intention is to consider the enhancement of the mixing caused by this interaction when compared to a noninteraction case. To observe the mixing that occurs, we examine Lagrangian particles (idealized fluid parcels) and how they disperse with time. These particles are initiated close together in a small area in the jet, and their trajectories followed by integrating the velocity field forward in time. The calculated trajectories for the jet-eddy interaction compare more favorably with observations than the trajectories for the no-eddy case.

\section{Analytical motivation and numerical experiments}

The model discussed by Bower (1991) is a simple two-dimensional jet that propagates eastward with constant phase speed and invariant shape. Consider a similar jet, given by the streamfunction in $x$ (westeast) and $y$ (north-south) fixed coordinates:

$\psi_{\text {jet }}(x, y, t)=J \tanh \left\{\frac{1}{L}(y-A \sin [k(x-c t)]\}\right.$,

where

$$
\begin{array}{ll}
J & \text { is the amplitude of the jet's streamfunction } \\
L & \text { is a factor controlling the jet's width } \\
A, k, c & \begin{array}{l}
\text { are the meander amplitude, wavenumber, } \\
\text { and phase speed, respectively. }
\end{array}
\end{array}
$$

Figure la shows this streamfunction over two wavelengths for parameters typical of the Gulf Stream. The meanders have a wavelength of $400 \mathrm{~km}$, an amplitude of $60 \mathrm{~km}$, a propagation speed of $10 \mathrm{~km} \mathrm{day}^{-1}$, and the jet width is $100 \mathrm{~km}$. The amplitude $J$ in Eq. (1) is chosen such that the maximum speed in the jet is about $150 \mathrm{~cm} \mathrm{~s}^{-1}$. Figure $1 \mathrm{a}$ also shows the velocity vectors composed of $u$ (the west-east component) and $v$ (the north-south component), where

$$
\begin{aligned}
& u=-\frac{\partial \psi}{\partial y} \\
& v=\frac{\partial \psi}{\partial x} .
\end{aligned}
$$

The trajectories of particles launched in the jet have been discussed, in some detail, by Bower (1991) and Dutkiewicz et al. (1993). As a brief summary it is instructive to consider Fig. 1b, which shows the streamfunction in moving coordinates, $X=x-c t$ and $Y=y$ :

$$
\Psi_{\text {jet }}(X, Y)=J \tanh \left\{\frac{1}{L}[Y-A \sin (k X)]\right\}+c Y .
$$
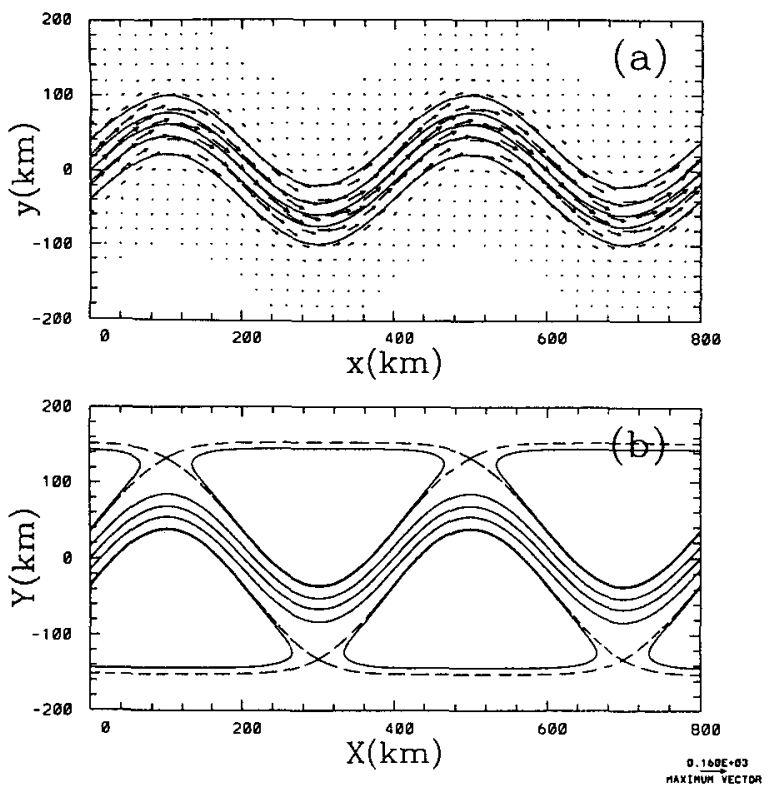

FIG. 1. (a) No-eddy interaction: streamfunction, $\psi_{\text {jet }}$, in the fixed reference frame $x, y$. Arrows are velocity vectors (in $\mathrm{cm} \mathrm{s}^{-1}$ ); (b) No eddy interaction: streamfunction, $\Psi_{\text {jet }}$, in the moving reference frame $X, Y$. Dashed lines show the separatrices.

Since $\Psi_{\mathrm{jet}}(X, Y)$ is time independent, particles will remain on streamlines (see Fig. 1b). Particles deployed in the jet will exhibit one of two possible trajectories: In the first they follow open streamlines, progressing downstream faster than the phase speed (particles deployed in the jet core); in the second they remain trapped in the meander crests or troughs, the closed streamlines, and travel downstream with average speed of $c$ (particles deployed in the recirculation regions). The two regions in the jet are bounded by the separatrices (shown as dashed lines in Fig. 1b). A third region is the far-field region to the north and south of the jet where fluid parcels are not affected by the jet. Since parcels will not leave the streamline in the moving $X, Y$ coordinates, they can exhibit only periodic trajectories.

To introduce deterministic mixing across the boundaries separating the three types of regions in this system, particles must be allowed to leave the streamlines shown in Fig. 1b. In this study we accomplish this by letting the jet interact with another mesoscale feature. Since the Gulf Stream does frequently interact with eddies, which have been observed to enhance float escape from the stream (Bower and Rossby 1989), we consider as a simplest case the effects of our jet interacting with a single stationary eddy. The eddy streamfunction at the fixed position $(x, y)$ is given as a simple Gaussian:

$$
\begin{aligned}
& \psi_{\text {eddy }}(x, y) \\
& \quad=E \exp \left[-\left(x-\mu_{x}\right)^{2} / 2 \sigma^{2}-\left(y-\mu_{y}\right)^{2} / 2 \sigma^{2}\right],
\end{aligned}
$$



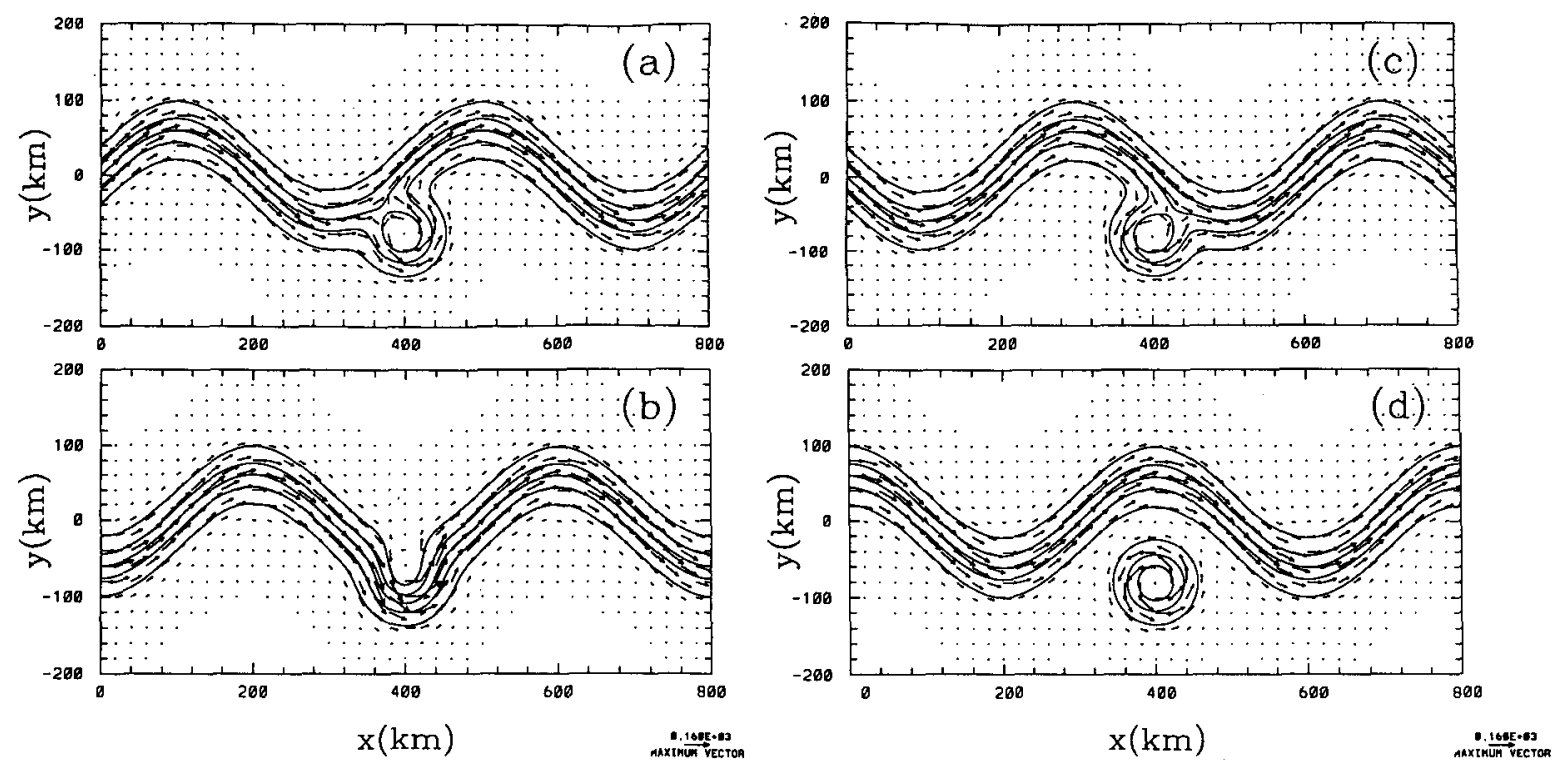

FIG. 2. Jet-eddy interaction for day (a) 0, (b) 10, (c) 20, and (d) 30 . (Note that day 40 will be same as day 0 .) Lines are streamfunction, $\psi_{\text {system }}$, in fixed reference frame $x, y$. Arrows are velocity vectors $\left(\right.$ in $\left.\mathrm{cm} \mathrm{s}^{-1}\right)$.

$E$

scaling factor determining maximum velocity

$\left(\mu_{x}, \mu_{y}\right)$ the position of the center of the eddy

$\sigma^{2}$ the variance of the Gaussian.

Although the eddy described in Eq. (4) perturbs the velocity field everywhere, the perturbation is exponential and even $100 \mathrm{~km}$ from the eddy's center is negligible.

The entire system to be considered will then be (in fixed coordinates $x$ and $y$ )

$$
\psi_{\text {system }}(x, y, t)=\psi_{\text {jet }}(x, y, t)+\psi_{\text {eddy }}(x, y),
$$

and can be seen in Fig. 2 with the eddy parameters of a radius (i.e., $e$-folding distance) of $50 \mathrm{~km}$ and a maximum speed of $150 \mathrm{~cm} \mathrm{~s}^{-1}$, similar to the maximum speed of the jet. The eddy is positioned with its center at fixed coordinates, $(x, y)=(400,-80)$. We have made the simplification that the eddy is stationary, but note that for an eddy propagating westward our results would be valid in a coordinate system moving with the eddy.

We will study the mixing process by looking at the dispersion of particles placed in a small bin within the jet. The mixing effect will be seen most efficiently for particles launched close to a separatrix, since from general principles of nonlinear dynamic systems (Ottino 1989), particles from this position will be more likely to move from one region to another when the system is perturbed. The position chosen in this study was in the crest of the jet, in the recirculation region, but close to the separatrix. (Many other positions would have given us as good, or possibly better, demonstration of the mixing, and many more would have given less dramatic cases.) The center of the particle bin, in fixed coordinates, is at $(x, y)=(100,32)$, and the bin is $(5$ $\mathrm{km})^{2}$. The size of the bin is arbitrary, but was chosen to give a good statistical basis. As the bin size is arbitrary we cannot quantify the mixing that occurs, but the results can be used to compare different experiments that use the same size bin. Nine hundred particles were launched from this bin in each of two experiments. In the first experiment, the jet is not perturbed (Fig. 1a), in the second experiment the jet is perturbed by the eddy discussed above (Fig. 2). In the following, we discuss the results of and, in particular, the differences between the two experiments. Particle trajectories are integrated for 80 days (i.e., two meander periods). The idealized, elastic collision (Fig. 2 ) will be vastly different in the real ocean where other perturbations (e.g., $\beta$ effect and other sources of potential vorticity perturbations) will induce phase changes into this idealized collision. There are, however, other studies in which this nearly elastic collision is anticipated (Spall and Robinson 1990).

Figures 3 and 4 show particles positions in $X$ and $Y$ at days $20,40,60$, and 80 for the no-eddy case and the eddy case, respectively. In the former, the particles remain trapped in the recirculation region in which they were deployed; however, they do spread out within the region due to the spatial variation in the jet's velocity and the slight differences in the particles' launch positions. Figure 4 shows the dramatic difference in the position of particles when the jet interacts with an eddy. At day 20, some particles have moved out of the recirculation region in which they were initially deployed, and some particles are seemingly unaffected by the eddy (those in the southern part of the initial recirculation region). By day 40, particles are in three recirculation regions: the initial recirculation region and the one up- 


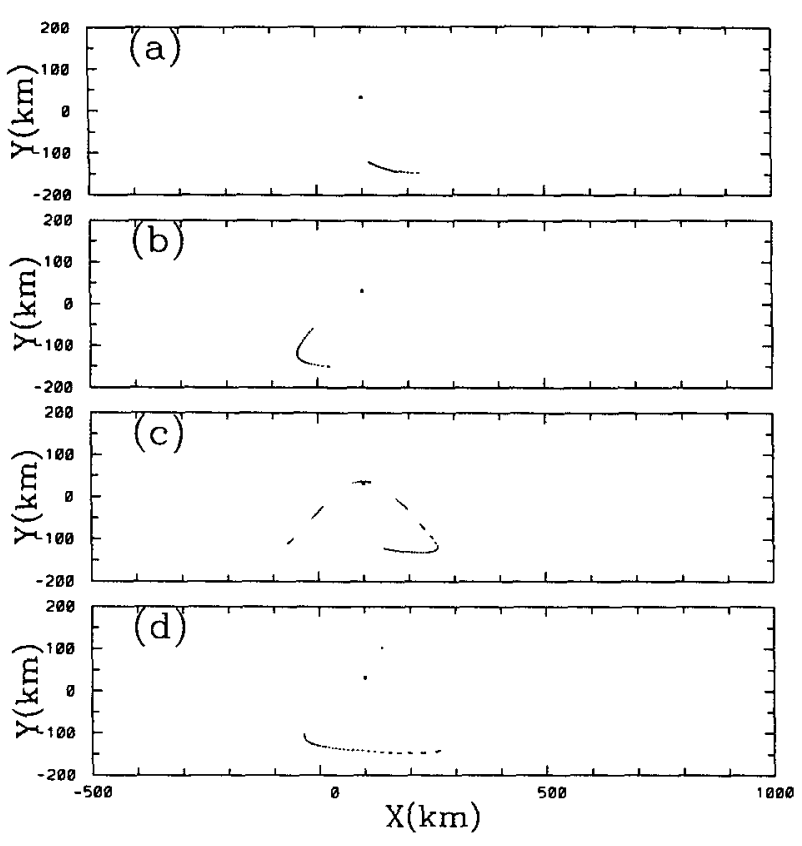

FIG. 3. No jet-eddy interaction: position of particles in moving coordinates $X, Y$ at day (a) 20, (b) 40, (c) 60, and (d) 80. Particles were launched from within the box shown at $(100,32)$.

stream and downstream of it, on the southern side of the jet. By day 60 it is evident that mixing is not only occurring between regions, but the mixing within regions is also enhanced (indicated by the folding seen in the initial recirculation region).

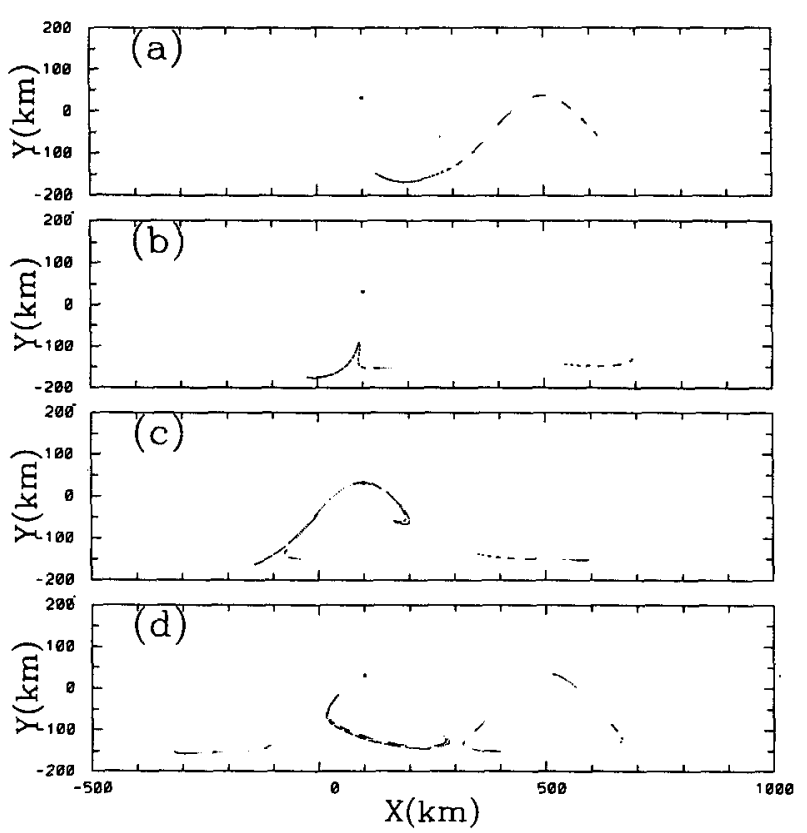

Fig. 4. Jet-eddy interaction: position of particles in moving coordinates $X, Y$ at day (a) 20, (b) 40, (c) 60, and (d) 80. Particles were launched from within the box shown at $(100,32)$.

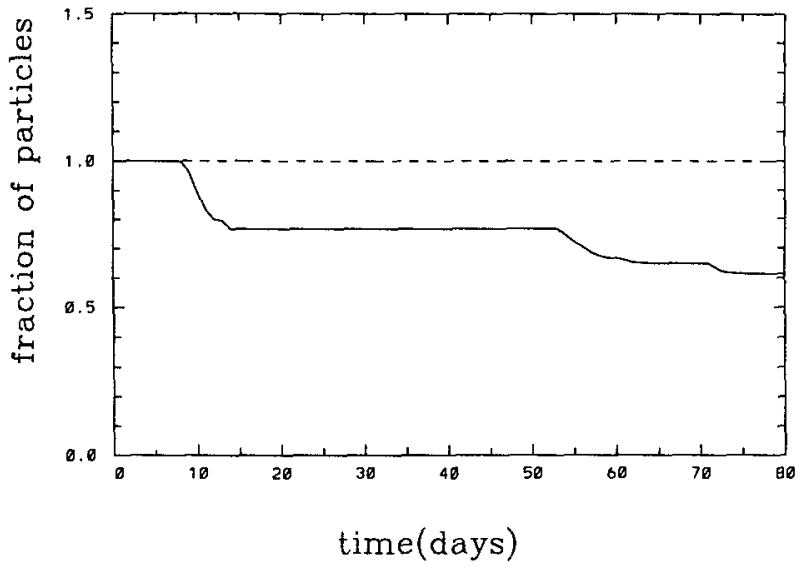

FIG. 5. Fraction of particles left in the initial recirculation region. Dashed line shows fraction for no-eddy case; solid line shows eddy interaction case.

A time series of the fraction of the 900 particles remaining in the initial recirculation region is shown in Fig. 5. The "steps" in this figure show that the escape of particles into other regions of the jet due to the interaction with the eddy does not take place continuously. Instead, this escape of particles from the initial region as well as the interaction of a meander with the eddy occur at intermittent intervals.

Figure 6 shows the mean-square dispersion of particles in both no-eddy and eddy cases. These dispersion curves are computed by monitoring the evolution of the average distance between 300 randomly chosen pairs of particles. In the no-eddy case, dispersion increases as particles mix within the recirculation region in which they were launched. This mixing is a consequence of their slightly different

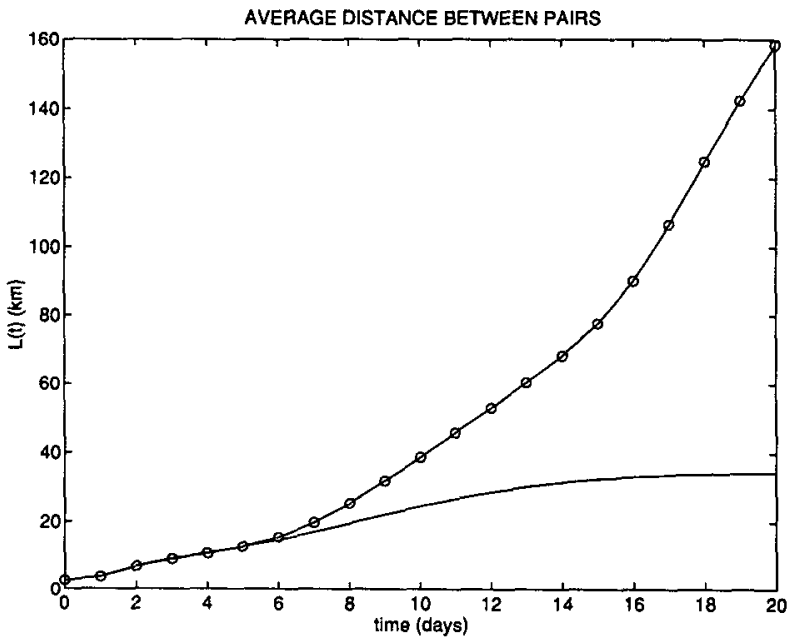

FIG , 6. Average distance between pairs. Line with circles shows exponential curve ( $e$-folding time of approximately 6 days) for experiment with jet-eddy interaction; solid line is curve for experiment with no jet-eddy interaction. 
launch positions, which results in slightly different propagation velocities. In the case of the jet-eddy interaction, however, the dispersion (at least for the first 20 days) is exponential with $e$-folding time of about 6 days.

\section{Discussion and summary}

In this study we have considered a very simplified two-dimensional model of a meandering jet that, when it interacts with a stationary eddy, causes exponential dispersion of particles launched in a bin near a separatrix in the jet. Obviously, we chose a position that would show a marked increase in dispersion when the jet interacted with the eddy; a somewhat less specific case of dispersion of fluid parcels in the jet would require a more global launching of particles or employing techniques such as the Melnikov function, as used by Samelson (1992). As an investigation of the importance of the initial conditions, an experiment was performed where a meridional section of particles was set off across the crest of the jet $(y=100 \mathrm{~km})$. When there is no eddy interaction, this meridional line is broken into two sections only-one in the open streamline region and one in the initial recirculation region [i.e., on either side of the separatrix (see Fig. 1b)]. When the jet interacts with the eddy, however, the same line stretches and breaks into several segments: particles further to the north on the meridional line move downstream in the open streamline region and are minimally affected by the eddy. Those particles close to either side of the separatrix move across the regions' boundaries as the line contorts. Particles in the recirculation region, away from the separatrix, are affected by the eddy field more intensely, and although they remain in the initial recirculation region, the line is dramatically more twisted and contorted than in the no-eddy case (Fig. 7).

The effect of the jet-eddy interaction vastly enhanced mixing within the jet system. Not only were particles now able to move across the separatrix and into different regions, but mixing within regions was greater. Obviously, the mixing would be even further enhanced if the jet interacted with more than one eddy.

Ottino (1989) states that time-independent two-dimensional flows are poor at mixing. We found that our unperturbed jet with dependence on only $x-c t$ and $y$, is indeed poor at mixing (demonstrated by small dispersion in Fig. 6). On the other hand, time-dependent flows should be good mixers of fluid parcels, with the possibility of becoming chaotic. Perturbations on otherwise predictable trajectories can cause chaos (e.g., Paldor and Boss 1992). In our case, the addition of an eddy, which perturbs the jet, allows for three degrees of freedom in the system $(x, y$, and $t)$, the minimum number for which chaos can occur (Pierrehumbert 1991). We do not prove that our trajectories are cha-
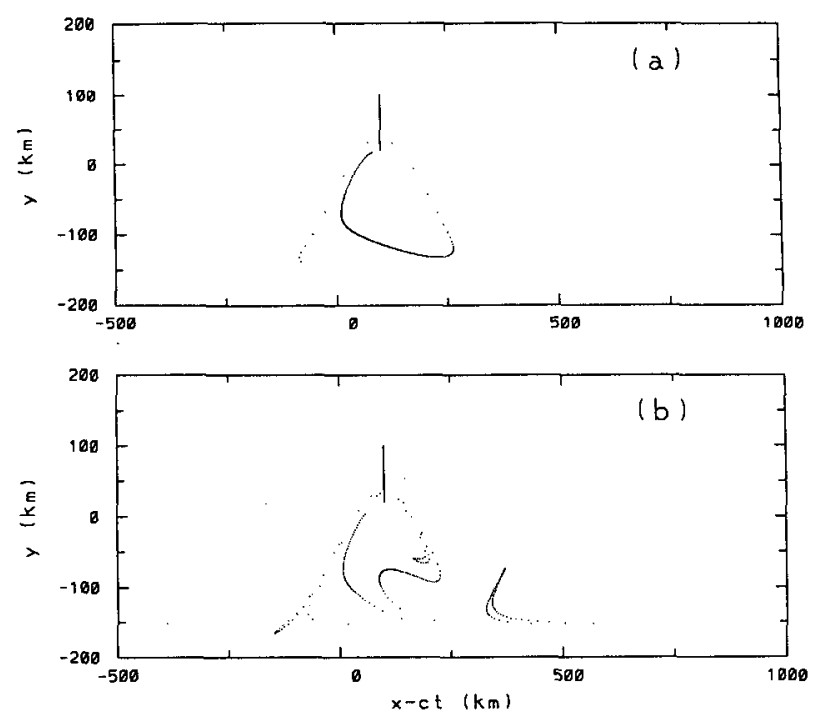

FIG. 7. Meridional section experiment: particles were launched from a meridional line. After 60 days the line is broken into two segments for no-eddy case (a) (the second segment is off the scale of the plot at this time as these particles move fast downstream); (b) when the eddy interacts with the jet several segments of contorted lines are encountered (in this case too, one segment of fast moving particles is off the scale of the plot).

otic, but the folding-stretching patterns seen in Fig. 4 and the exponential dispersion (Fig. 6) are certainly reminiscent of chaos. The importance of the ring-current interaction mechanism was also pointed out by Samelson (1992), who studied the effect of the meander amplitude modulation on the subsequent mixing. The suggestion was, however, not substantiated or quantified in Samelson's study by a specific model or calculation as in the present work.

Our findings on the enhancement of mixing due to the interaction of a meandering jet with an eddy has been validated observationally in the detailed survey of 37 floats launched in the Gulf Stream in 1984/85 reported by Bower and Rossby (1989) (Fig. 8a). They found that "the meander-induced cross-frontal fluid exchange is enhanced by ring-current interaction." Though, not quantified, this observation does lend credence to our calculation of the nearly five-fold increase in the rate of dispersal of fluid parcels when the eddy interacts with the meandering jet. But the correspondence between the observations and our model go beyond these heuristic findings. In Figure $8 \mathrm{~b}$ we show 40 particle trajectories in the no-eddy case. These trajectories give a good first approximation of float trajectories in the Gulf Stream (Fig. 8a), especially in the west, where distinctive meander-induced motion is seen. However, they are too regular and do not disperse enough downstream. For a second approximation, we consider the eddy-perturbed trajectories (Fig. 8c). These trajectories show much less regularity and a pronounced increase in downstream dispersion. Our goal in this experiment was to use known results from non- 

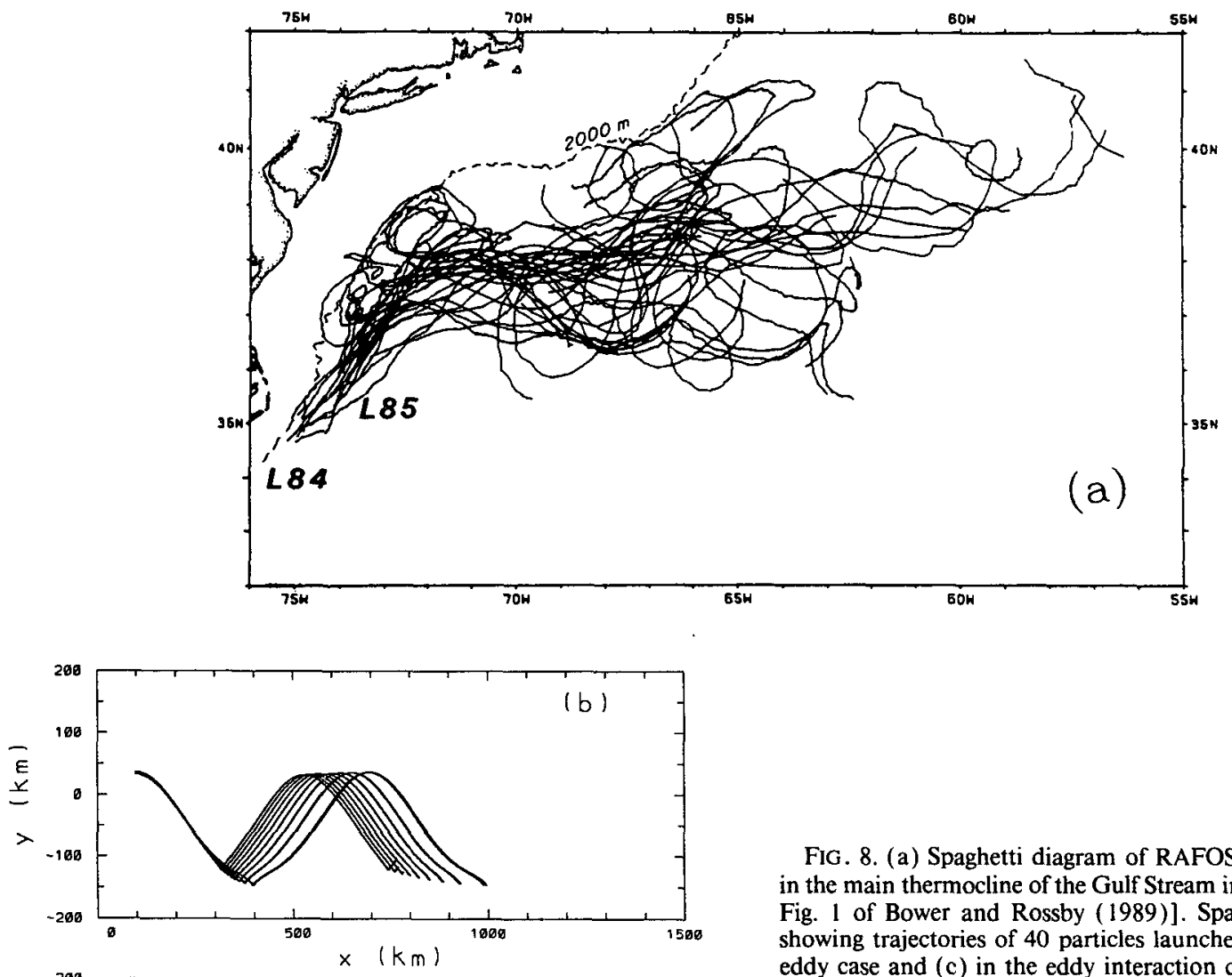

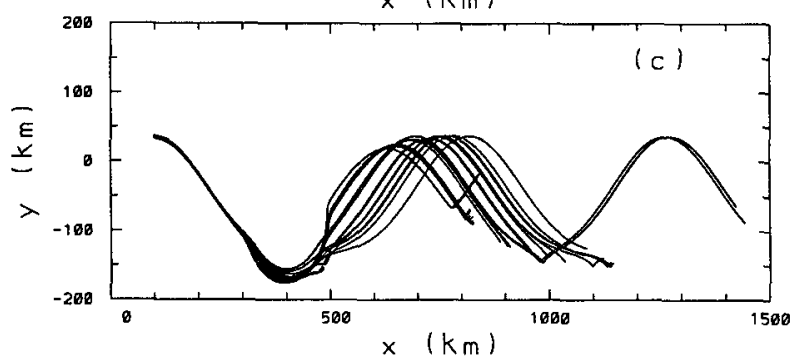

linear dynamics to modify an existing oceanographic dispersion model to better simulate observations: the results in Fig. 8c indicate progress has been achieved.

Acknowledgments. Helpful discussions with $\mathrm{M}$. Wimbush are gratefully acknowledged. NP would like to thank the hospitality extended to him at the Graduate School of Oceanography, University of Rhode Island, during his Sabbatical there and the support of ONR Grant N0001490J1602 to URI. SD would like to thank the Friends of Oceanography Organization at GSO/URI for their support. We also thank the reviewers for their instructive comments.

\section{REFERENCES}

Bower, A. S., 1991: A simple kinematic mechanism for mixing fluid parcels across a meandering jet. J. Phys. Oceanogr., 21, 173180.

- , and H. T. Rossby, 1989: Evidence of cross-frontal exchange
FIG. 8. (a) Spaghetti diagram of RAFOS floats launched in the main thermocline of the Gulf Stream in 1984/85 [from Fig. 1 of Bower and Rossby (1989)]. Spaghetti diagrams, showing trajectories of 40 particles launched in (b) the noeddy case and $(c)$ in the eddy interaction case. Trajectories are shown for 80 days. Many of the rigid constraints on the flow in the no-eddy case are removed when the eddy is introduced. processes in the Gulf Stream based on isopycnal RAFOS float data. J. Phys. Oceanogr., 19, 1177-1190.

Dutkiewicz, S., A. Griffa, and D. Olson, 1993: Particles diffusion in a meandering jet. J. Geophys. Res., 98, 16 487-16 500

Dewar, W. K., and G. R. Flierl, 1985: Particle trajectories and simple models of transport in coherent vortices. Dyn. Atmos. Oceans, 9, 215-252.

Nof, D., 1986: The collision between the Gulf Stream and warmcore rings. Deep-Sea Res., 33, 359-378.

Olson, D. B., 1991: Rings in the ocean. Ann. Rev. Earth Planet. Sci., 19, 283-311.

Ottino, J. M., 1989: The Kinematics of Mixing: Stretching, Chaos, and Transport. Cambridge University Press, $364 \mathrm{pp}$.

Paldor, N., and E. Boss, 1992: Chaotic trajectories of tidally perturbed inertial oscillations. J. Atmos. Sci., 49, 2306-2318.

Pierrehumbert, R. T., 1991: Chaotic mixing of tracer and vorticity by modulated travelling Rossby waves. Geophys. Astrophys. Fluid Dyn., 58, 285-319.

Samelson, R. M., 1992: Fluid exchange across a meandering jet. $J$. Phys. Oceanogr., 22, 431-440.

Spall, M. A., and A. R. Robinson, 1990: Regional primitive equation studies of the Gulf Stream meander and ring formation region J. Phys. Oceanogr., 20, 985-1016. 